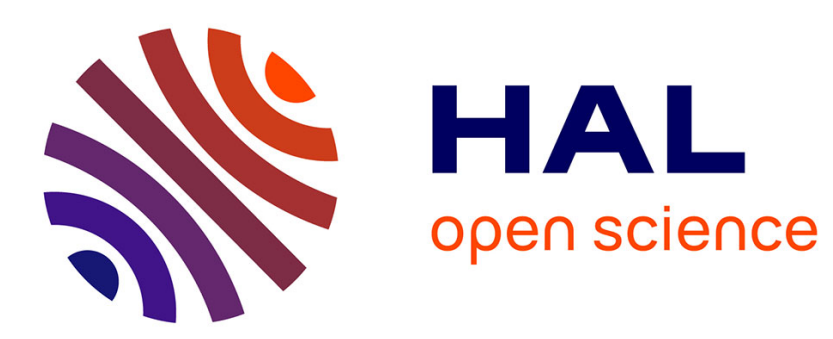

\title{
On the Cauchy problem for dispersive equations with nonlinear terms involving high derivatives and with arbitrarily large initial data
}

Thierry Colin

\section{- To cite this version:}

Thierry Colin. On the Cauchy problem for dispersive equations with nonlinear terms involving high derivatives and with arbitrarily large initial data. Nonlinear Analysis: Theory, Methods and Applications, 1994, 22 (7), pp.835-845. hal-00848097

\section{HAL Id: hal-00848097 \\ https://hal.inria.fr/hal-00848097}

Submitted on 25 Jul 2013

HAL is a multi-disciplinary open access archive for the deposit and dissemination of scientific research documents, whether they are published or not. The documents may come from teaching and research institutions in France or abroad, or from public or private research centers.
L'archive ouverte pluridisciplinaire HAL, est destinée au dépôt et à la diffusion de documents scientifiques de niveau recherche, publiés ou non, émanant des établissements d'enseignement et de recherche français ou étrangers, des laboratoires publics ou privés. 


\title{
On the Cauchy problem for dispersive equations with nonlinear terms involving high derivatives and with arbitrarily large initial data
}

\author{
Thierry COLIN* \\ Centre de Mathématiques et Leurs Applications \\ Ecole Normale Supérieure de Cachan \\ 61 Avenue du Président Wilson \\ 94235 CACHAN Cedex, France
}

\begin{abstract}
The aim of this article is to study the Cauchy problem for general nonlinear dispersive equations involving derivatives in the nonlinearity. The use of some decay properties of the linear part allows us to address the case of arbitrarily large initial data.
\end{abstract}

\section{Hypotheses and statement of the result}

\subsection{The evolution equation}

The goal of this work is to study the Cauchy problem for equations of the form

$$
i u_{t}+L u=F(u),
$$

where $u: \mathbb{R} \times \mathbb{R}^{n} \rightarrow \mathbb{C}$ and $L$ is a linear (pseudo -) differential operator of order $m$ with real valued symbol denoted by $l(\xi)$, and $F$ is a nonlinear, possibly nonlocal operator. We will only consider the case where the linear part of (1) is dispersive, i.e. $l(\xi) \neq C \xi$. Actually, we will address cases where the linear group $e^{i t L}$ satisfies some "decay" properties, see Section 1.2 below.

The nonlinear term in (1) will be of the following form :

$$
F(u)=\sum_{j=1}^{p} \mathcal{L}_{j}\left(f_{j}(u)\right)
$$

where $\mathcal{L}_{j}$ is a pseudo-differential operator with constant coefficients of order $l_{j}$ and $f_{j}: \mathbb{C} \rightarrow \mathbb{C}$ is smooth in the following sense: $f_{j}\left(u_{1}+i u_{2}\right)=g_{j}\left(u_{1}, u_{2}\right)$, with $g_{j} \in \mathcal{C}^{r}\left(\mathbb{R}^{2}, \mathbb{R}^{2}\right)$.

*This work was partially supported by the Ministère de la Recherche et de la Technologie, France, Contrat N ${ }^{0} 90 \mathrm{~S} 0315$ 


\subsection{Hypotheses}

In what follows, we denote by $H^{s}$ and $W^{s, p}$ the Sobolev spaces $H^{s}\left(\mathbb{R}^{n}\right)$ and $W^{s, p}\left(\mathbb{R}^{n}\right)$. We write now precisely our hypotheses on $\mathcal{L}_{j}, f_{j}$ and $L$ :

- (H1) we suppose that there exists $m \geq 2$, such that $e^{i L t} \equiv W_{0}(t)$, which is a group on every Sobolev space $H^{s}\left(\mathbb{R}^{n}\right)$, satisfies : for every $0 \leq \theta<\frac{2}{n}$ (in the case where $n=1$, we impose $0 \leq \theta \leq 1)$, denoting by $(q, p)$ the pair $\left(\frac{4}{n \theta}, \frac{2}{1-\theta}\right)$ and by $\left(q^{\prime}, p^{\prime}\right)$ the conjugate exponents, we have the following estimates, for every $T \in \mathbb{R}$,

$$
\begin{gathered}
(H 1)-1 \quad\left|W_{0}(t) u_{0}\right|_{L^{q}\left(0 ; T ; W^{\frac{\theta}{4} n(m-2), p}\right)} \leq M(|T|)\left|u_{0}\right|_{L^{2}}, \\
(H 1)-2\left|\int_{0}^{t} W_{0}(t-\tau) g(\cdot ; \tau) d \tau\right|_{L^{q}\left(0 ; T ; W^{\frac{\theta}{2} n(m-2), p}\right)} \leq M(|T|)|g|_{L^{q^{\prime}\left(0 ; T ; L^{p^{\prime}}\right)}}, \\
(H 1)-3 \quad\left|\int_{0}^{t} W_{0}(t-\tau) g(\cdot ; \tau) d \tau\right|_{L^{\infty}\left(0 ; T ; L^{2}\right)} \leq M(|T|)|g|_{L^{q^{\prime}\left(0 ; T ; L^{p^{\prime}}\right)}},
\end{gathered}
$$

where $M$ is a non decreasing function from $\mathbb{R}^{+}$into $\mathbb{R}^{+}$.

- (H2) The second hypothesis is on the operators $\mathcal{L}_{j}$ (we denote by $\widehat{\mathcal{L}_{j}}$ their symbols) :

$$
\left|D^{\alpha} \widehat{\mathcal{L}_{j}}(\xi)\right| \leq C(1+|\xi|)^{l_{j}-|\alpha|} \text { with } l_{j}<\frac{m-2}{2} \text { and }|\alpha|<\left[\frac{n}{2}\right]+1
$$

Remark 1 : Thanks to Hörmander's multiplier's Theorem (see Torchinsky [11]), it is clear that $\mathcal{L}_{j}(I-\Delta)^{-l_{j} / 2}$ maps $L^{p}$ into $L^{p}$ continuously for all $1<p<\infty$. We will use this fact without notice in the course of the proof of Theorem 1.

We now denote by $l=\max _{j=1, \ldots, p} l_{j}$.

- (H3) The third hypothesis is on the nonlinearity :

$\exists s \geq 0, \sigma_{j}>0$ and $0 \leq \theta<\frac{2}{n}$ (in the case where $n=1$, we impose $0 \leq \theta \leq 1)$ such that

$$
\begin{gathered}
\left|f_{j}(u)\right|_{W^{s+l, \beta^{\prime}}} \leq C|u|_{H^{s}}^{\sigma_{j}}|u|_{W^{s+k, \beta}}, \\
\left|f_{j}(u)-f_{j}(v)\right|_{L^{\beta^{\prime}}} \leq C\left(|u|_{H^{s}}^{\sigma_{j}}+|v|_{H^{s}}^{\sigma_{j}}\right)|u-v|_{L^{\beta}},
\end{gathered}
$$

with $k=\frac{\theta n(m-2)}{4}, \theta=1-\frac{2}{\beta}$ and $\frac{1}{\beta}+\frac{1}{\beta^{\prime}}=1$.

With the previous hypotheses and notations, our main result reads as follows. 


\section{Theorem 1}

- Under (H1), (H2), (H3), if $u_{0} \in H^{s}$ then (1) has an unique maximal solution on $\left[0 ; T\left(u_{0}\right)\left[\right.\right.$ in $\mathcal{C}\left(\left[0 ; T\left(u_{0}\right)\left[; H^{s}\right)\right.\right.$. Moreover $u \in L^{q}\left(0 ; t ; W^{k+s, \beta}\right)$ for

$$
\frac{2}{q}=n\left(\frac{1}{2}-\frac{1}{\beta}\right), \quad \forall t<T\left(u_{0}\right)
$$

- $u(t)$ depends continuously on $u_{0}$ in the following sense : if $u_{0}^{n} \rightarrow u_{0}$ in $H^{s}$, then $\forall T<T\left(u_{0}\right)$, if $n$ is sufficiently large $u^{n}(t)$ exist on a common interval $[0 ; T]$ and $u^{n}(t) \rightarrow u(t)$ in $\mathcal{C}\left([0 ; T] ; H^{s}\right) \cap L^{q}\left(0 ; T ; W^{k+s, \beta}\right)$.

Remark 2 : It is well known that for some equations $T\left(u_{0}\right)<+\infty$, (even for semilinear equations with $F(u)=-|u|^{2} u$ and $L=\Delta$ ) see for example Glassey [3].

\subsection{Comments on (H1), (H2), and (H3)}

In [4] Kenig, Ponce and Vega prove that if the symbol of $L$ is an elliptic polynomial, or if it is tensorial, then $L$ satisfies (H1).

In section 2 below, we show that if the functions $f_{j}$ are $\mathcal{C}^{r}$ with $r \geq[k]+1$ and if there exists $\sigma_{j} \geq \frac{8 l}{n(m-2)}$ such that

$$
\forall \alpha \text { with }|\alpha| \leq r,\left|\left(D^{\alpha} f_{j}\right)(u)\right| \leq c_{\alpha, j}|u|^{\sigma_{j}+1-|\alpha|},
$$

then $f_{j}$ satisfies $(\mathrm{H} 3)$.

The estimate (2) means that $f_{j}$ and its derivatives behave like a power of $u$, so that Theorem 1 applies.

For example, we have :

Theorem 2 The problem

$$
\begin{cases}i u_{t}+(-\Delta)^{a} u & =\sum_{j=1}^{p} a_{j} D^{l_{j}}\left(|u|^{\sigma_{j}} u\right) \\ u(x ; 0) & =u_{0}(x)\end{cases}
$$

with $l=\max l_{j}<a-1, \sigma_{j} \geq \frac{4 l}{n(a-1)}$ is locally well-posed in $H^{s}$ for $s \geq$ $n\left[\frac{1}{2}-\frac{2 l}{n(a-1) \sigma}\right]$ with $\sigma=\max \sigma_{j}$.

Some results on the Cauchy problem for dispersive equations are available in the litterature; in [5], Kenig, Ponce, Vega prove that $\frac{\partial u}{\partial t}+\partial_{x}\left(u^{k}\right)+\partial_{x^{3}} u=0$ is locally well posed in $H^{s}$ for $s$ depending on $k$. In dimension $n \geq 2$, they show in [6] that $\partial_{t} u=i \Delta u+P\left(u ; P_{x} u ; \bar{u} ; \nabla_{x} \bar{u}\right)$ where $P$ is a complex polynomial is well posed in some weighted Sobolev spaces for small initial data. There exists another result (Klainerman-Ponce [7]) for $i u_{t}-\Delta u=F(u ; \nabla u)$ in $\mathbb{R}^{n}$ for small initial data in $H^{s}, s>\frac{n}{2}+2$ under restrictive hypotheses on the form of $F$. 
The difference with our work is that we do not impose to the initial data to be small, and we work in the spaces $H^{s}\left(\mathbb{R}^{n}\right)$ which are the natural spaces corresponding to the linear part. Actually, our results are more restrictive on the nonlinearity since we consider general situations, and therefore, we do not have a large variety of estimates on the linear group, as it is the case for the Airy equation which is the linear part of the $\mathrm{KdV}$ equation [5].

We can compare our results with the work of J.C. Saut [9]. He proves that $\frac{\partial u}{\partial t}+\sum_{i=1}^{n} \partial_{x_{i}}[f(t, u)+L(x ; u)]=g(x ; t)$ is well posed, where $L$ is an elliptic operator and $f(t, u)$ a polynomial in $u$ which maximal degree depends on $L$. The method used in our paper gives a slightly different result. Indeed, the degree of $f$ is not limited, but we replace $\frac{\partial}{\partial x_{i}}$ by a pseudo-differential operator whose order is limited by that of $L$. Moreover our result holds for non elliptic operators as long as they satisfy (H1).

\section{Examples of nonlinearities satisfying (H3)}

Suppose that for $r \geq[k]+1, f_{j}$ is $\mathcal{C}^{r}$ and that there exists $\sigma_{j} \geq \operatorname{Max}\left(\frac{8 l}{n(m-2)}, r-1\right)$ such that

$$
\forall \alpha \text { with }|\alpha| \leq r \text { then }\left|D^{\alpha} f_{j}(u)\right| \leq C_{\alpha, j}|u|^{\sigma_{j}+1-|\alpha|} .
$$

We have :

Proposition 1 : If $u \in W^{s+l, \beta} \cap E^{z_{j}}$, then $f_{j}(u) \in W^{s+l, \beta^{\prime}}$, with $\frac{1}{\beta^{\prime}}=\frac{\sigma_{j}}{z_{j}}+\frac{1}{\beta}$ for $+\infty>\beta>1, z_{j}>1$ and

$$
\left|f_{j}(u)\right|_{W^{s+l, \beta^{\prime}}} \leq c|u|_{L^{z_{j}}}^{\sigma}|u|_{W^{s+l, \beta}}
$$

This kind of inequality is essentially due to Y. Meyer [8] for the case $z_{j}=$ $+\infty$. Christ and Weinstein in [2] prove a related result :

$$
\begin{gathered}
\left|D^{\alpha} F(u)\right|_{L^{p}} \leq C\left|F^{\prime}(u)\right|_{L^{q}}\left|D^{\alpha} u\right|_{L^{r}} \\
\frac{1}{p}=\frac{1}{q}+\frac{1}{r}, \quad 0<\alpha<1 \text { and } u: \mathbb{R} \rightarrow \mathbb{R} .
\end{gathered}
$$

We give the proof of Proposition 1 in the Appendix.

Now we prove that $f_{j}$ satisfies (H3).

Let $\sigma=M a x \sigma_{j}$, take $\theta$ given by $l=\frac{\theta n(m-2)}{4}$ (recall that $l=M_{a x} l_{j}$ ) and $z$ given by $\theta=\frac{\sigma}{z}$.

Choose $z_{j}$ such that $\frac{\sigma_{j}}{z_{j}}=\frac{\sigma}{z}$ ie $z_{j}=\frac{z \sigma_{j}}{\sigma} \leq z$. And since we impose the condition $\sigma_{j} \geq \frac{8 l}{n(m-2)}$, we have $z_{j} \geq 2$.

We now choose $s$ such that $H^{s} \hookrightarrow L^{z}$ and since $2 \leq z_{j} \leq z, H^{s} \hookrightarrow L^{z_{j}}$ so that the proposition implies that (H3) is satisfied. The application of this fact and of Theorem 1 prove Theorem 2. 


\section{Proof of Theorem 1}

First we transform (1) :

$$
\begin{cases}i u_{t}+L u & =\sum_{j=1}^{p} \mathcal{L}_{j}\left(f_{j}(u)\right) \\ u(x ; 0) & =u_{0}(x)\end{cases}
$$

into the equivalent integral equation (we shall prove later on that they are equivalent, see Section 3.4 below).

$$
(I N T) \mathcal{T}(u) \equiv W_{0}(t) u_{0}-i \int_{0}^{t} W_{0}(t-s)\left(\sum_{j=1}^{p} \mathcal{L}_{j}\left(f_{j}(u)\right)(s)\right) d s=u(t) .
$$

\subsection{Some estimates on $\mathcal{T}(u)$ :}

Lemma $1 \mathcal{T}(u)$ satisfies

a)

$$
|\mathcal{T}(u)|_{L^{\infty}\left(0 ; T ; H^{s}\right) \cap L^{q}\left(0 ; T ; W^{k+s, \beta)}\right.}
$$

$$
\leq C_{1}(1+M(|T|))\left|u_{0}\right|_{H^{s}}+C_{2} M(|T|) T^{\delta}\left(\sum_{j=1}^{p}|u|_{L^{\infty}\left(0 ; T ; H^{s}\right)}^{\sigma_{j}}\right)|u|_{L^{q}\left(0 ; T ; W^{k+s, \beta}\right)},
$$

and

b)

$$
\begin{gathered}
|\mathcal{T}(u)-\mathcal{T}(v)|_{L^{q}\left(o ; T ; L^{\beta}\right)} \leq C_{3} M(|T|) T^{\delta}\left(\sum_{j=1}^{p}|u|_{L^{\infty}\left(0 ; T ; H^{s}\right)}^{\sigma_{j}}\right. \\
\left.+|v|_{L^{\infty}\left(0 ; T ; H^{s}\right)}^{\sigma_{j}}\right)|u-v|_{L^{q}\left(0 ; T ; L^{\beta}\right)}, \\
\text { where } \delta=\frac{1}{q^{\prime}}-\frac{1}{q}>0 .
\end{gathered}
$$

Proof : a) In order to estimate $\mathcal{T}(u)$ in $L^{\infty}\left(0 ; T ; H^{s}\right) \cap L^{q}\left(0 ; T ; W^{k+s, \beta}\right)$, we need to estimate two terms :

i) The linear term $W_{0}(t) u_{0}$ :

$$
\left|W_{0}(t) u_{0}\right|_{H^{s}}=\left|u_{0}\right|_{H^{s}}
$$

since $W_{0}(t)$ is unitary in $H^{s}$.

$$
\left|W_{0}(t) u_{0}\right|_{W^{k+s, \beta}} \leq C\left|W_{0}(t)(I-\Delta)^{s / 2} u_{0}\right|_{W^{k, \beta}},
$$

but since $k=\frac{\theta n(m-2)}{4}$ by (H1) -1 , we have

$$
\left|W_{0}(t) u_{0}\right|_{L^{q}\left(0 ; T ; W^{k,+s, \beta}\right)} \leq C M(|T|)\left|(I-\Delta)^{s / 2} u_{0}\right|_{L^{2}}=C\left|u_{0}\right|_{H^{s}} .
$$


Then (3) and (4) lead to

$$
\left|W_{0}(t) u_{0}\right|_{L^{\infty}\left(0, T, H^{s}\right) \cap L^{q}\left(0, T, W^{k+s, \beta}\right)} \leq C_{1}(1+M(|T|))\left|u_{0}\right|_{H^{s}}
$$

ii) The nonlinear term $\int_{0}^{t} W_{0}(t-s) \sum_{j=1}^{p} \mathcal{L}_{j}\left(f_{j}(u)\right)(s) d s$ :

We estimate each term separately :

- $\quad\left|\int_{0}^{t} W_{0}(t-s) \mathcal{L}_{j} f_{j}(u)(s) d s\right|_{L^{\infty}\left(0 ; T ; H^{s}\right)}$

$$
\begin{gathered}
\leq C\left|\int_{0}^{t} W_{0}(t-s)(I-\Delta)^{s / 2} \mathcal{L}_{j}\left(f_{j}(u)\right) d s\right|_{L^{\infty}\left(0 ; T ; L^{2}\right)}, \\
\quad \leq C M(|T|)\left|(I-\Delta)^{s / 2} \mathcal{L}_{j}\left(f_{j}(u)\right)\right|_{L^{q^{\prime}}\left(0 ; T: L^{\beta^{\prime}}\right)}
\end{gathered}
$$

by (H1)-3,

$$
\leq C M(|T|)\left|f_{j}(u)\right|_{L^{q^{\prime}\left(0 ; T ; W^{s+l_{j}, \beta_{j}}\right)}}
$$

by $(\mathrm{H} 2)$. So that

$$
\left|\int_{0}^{t} W_{0}(t-s) \mathcal{L}_{j} f_{j}(u)(s) d s\right|_{L^{\infty}\left(0 ; T ; H^{s}\right)} \leq C M(|T|) T^{\delta}|u|_{L^{\infty}\left(0 ; T ; H^{s}\right)}^{\sigma_{j}}|u|_{L^{q}\left(0 ; T ; W^{s+k, \beta}\right)}
$$

by (H3) and Hölder's inequality with respect to time.

- On the other hand :

$$
\begin{gathered}
\left|\int_{0}^{t} W_{0}(t-s) \mathcal{L}_{j}\left(f_{j}(u)\right)(s) d s\right|_{L^{q}\left(0 ; T ; W^{k+s, \beta}\right)} \\
\leq C\left|\int_{0}^{t} W_{0}(t-s)(I-\Delta)^{s / 2} f_{j}(u) d s\right|_{L^{q}\left(0 ; T ; W^{k+l_{j}, \beta_{j}}\right)}
\end{gathered}
$$

by (H2),

$$
\leq C M(|T|)\left|f_{j}(u)\right|_{L^{q^{\prime}}\left(0 ; T ; W^{s, \beta_{j}^{\prime}}\right)}
$$

by $(\mathrm{H} 1)-2$ since $k+l \leq \frac{\theta n(m-2)}{2}$,

$\left|\int_{0}^{t} W_{0}(t-s) \mathcal{L}_{j}\left(f_{j}(u)\right)(s) d s\right|_{L^{q}\left(0 ; T ; W^{k+s, \beta}\right)} \leq C M(|T|) T^{\delta}|u|_{L^{\infty}\left(0 ; T ; H^{s}\right)}^{\sigma_{j}}|u|_{L^{q}\left(0 ; T ; W^{s+k, \beta)}\right.}$

by (H3) and Hölder's inequality with respect to time; (5), (6) and (7) together give the estimate of the a) of Lemma 1.

b) $|\mathcal{T}(u)-\mathcal{T}(v)|_{L^{q}\left(0 ; T ; L^{\beta}\right)}=\left|\int_{0}^{t} W_{0}(t-s) \sum_{j=1}^{p} \mathcal{L}_{j}\left(f_{j}(u)-f_{j}(v)\right) d s\right|_{L^{q}\left(0 ; T ; L^{\beta}\right)}$

$$
\leq C M(|T|) \sum_{j=1}^{p}\left|f_{j}(u)-f_{j}(v)\right|_{L^{q^{\prime}}\left(0 ; T ; L^{\beta^{\prime}}\right)}
$$


by(H1)-2,

$$
\leq C M(|T|) T^{\delta} \sum_{j=1}^{p}\left(|u|_{L^{\infty}\left(0 ; T ; H^{s}\right)}^{\sigma_{j}}+|v|_{L^{\infty}\left(0 ; T ; H^{s}\right)}^{\sigma_{j}}\right)|u-v|_{L^{q}\left(0 ; T ; L^{\beta}\right)}
$$

by (H3) and Hölder's inequality with respect to time.

The proof of Lemma 1 is complete.

\subsection{Existence and uniqueness}

Now we fix $T_{1}>0$ and we consider $R^{\prime} \equiv C_{1}\left|u_{0}\right|_{H^{s}}\left(1+M\left(\left|T_{1}\right|\right)\right),\left(C_{1}\right.$ is the constant appearing in a) of Lemma 1).

Proposition 2 Let $R>R^{\prime}$, if $T$ is sufficiently small, then $\mathcal{T}$ maps the ball of radius $R$ in $L^{\infty}\left(0 ; T ; H^{s}\right) \cap L^{q}\left(0 ; T ; W^{k+s, \beta}\right)$ in itself and it is a contraction in the norm of $L^{q}\left(0 ; T ; L^{\beta}\right)$.

\section{Proof:}

- Indeed by a) of Lemma 1 , if $T \leq T_{1}$ then

$$
|\mathcal{T}(u)|_{L^{\infty}\left(0 ; T ; H^{s}\right) \cap L^{q}\left(0 ; T ; W^{k+s ; \beta}\right)} \leq R^{\prime}+C_{2} M(|T|) T^{\delta}\left(\sum_{j=1}^{p} R^{\sigma_{j}}\right) R .
$$

It follows that if $T$ is sufficiently small,

$$
|\mathcal{T}(u)|_{L^{\infty}\left(0 ; T ; H^{s}\right) \cap L^{q}\left(0 ; T ; W^{k+s, \beta}\right)} \leq R .
$$

- b) of Lemma 2 gives :

$$
|\mathcal{T}(u)-\mathcal{T}(v)|_{L^{q}\left(0 ; T ; L^{\beta}\right)} \leq C_{3} M(|T|) T^{\delta} 2 \sum_{j=1}^{p} R^{\sigma_{j}}|u-v|_{L^{q}\left(0 ; T ; L^{\beta}\right)}
$$

We take $T$ such that $C_{3} M(|T|) T^{\delta} 2 \sum_{j=1}^{p} R^{\sigma_{j}} \leq 1 / 2$, thereby proving the proposition.

Now we remark that a ball in $L^{\infty}\left(0 ; T ; H^{s}\right) \cap L^{q}\left(0 ; T ; W^{s+k, \beta}\right)$ is complete for the norm of $L^{q}\left(0 ; T ; L^{\beta}\right)$. Hence the contraction principle gives a local solution to (INT). Since $T$ depends only on $R$ which in its turn depends only on $\left|u_{0}\right|_{H^{s}}$, the existence time of the maximal solution depends only on $|u|_{H^{s}}$ i.e. : if $T\left(u_{0}\right)$ (= the existence time of the maximal solution) is finite then

$$
\lim _{t \rightarrow T\left(u_{0}\right)}|u(t)|_{H^{s}}=+\infty
$$

Now if $u, v$ are two solutions to (INT) in $\mathcal{C}\left(\left[0 ; T_{0}\right] ; H^{s}\right)$ then

$$
\forall T<T_{0},|u-v|_{L^{q}\left(0 ; T ; L^{\beta}\right)}=|\mathcal{T}(u)-\mathcal{T}(v)|_{L^{q}\left(0 ; T ; L^{\beta}\right)},
$$




$$
\leq C_{3} M(|T|) T^{\delta}\left(\sum_{j=1}^{p}|u|_{L^{\infty}\left(0 ; T ; H^{s}\right)}^{\sigma_{j}}+|v|_{L^{\infty}\left(0 ; T ; H^{s}\right)}^{\sigma_{j}}\right)|u-v|_{L^{q}\left(0 ; T ; L^{\beta}\right)}
$$

by Lemma 1 b).

Therefore taking $T$ sufficiently small :

$$
|u-v|_{L^{q}\left(0 ; T ; L^{\beta}\right)} \leq 1 / 2|u-v|_{L^{q}\left(0 ; T ; L^{\beta}\right)}
$$

and $u \equiv v$ on $[0 ; T]$, thereby proving local uniqueness.

We have proved :

Proposition 3 For all $u_{0} \in H^{s}$, there exists a unique maximal solution $u$ to $(I N T)$ in $\mathcal{C}\left(\left[0 ; T\left(u_{0}\right)\left[; H^{s}\right)\right.\right.$. Moreover $\forall t<T\left(u_{0}\right), u \in L^{q}\left(0 ; t ; W^{k+s, \beta}\right)$.

\subsection{Continuous dependence with respect to the initial data}

Let $u_{0} \in H^{s}$ and $u_{0}^{n} \in H^{s}$ with $u_{0}^{n} \rightarrow u_{0}$ in $H^{s}$. We note

$$
\left.\mathcal{T}_{n}(v(t)) \equiv W_{0}(t) u_{0}^{n}-i \int_{0}^{t} W_{0}(t-s) \sum_{j=1}^{p} \mathcal{L}_{j}\left(f_{j}(v)\right) d s\right) .
$$

We call $u^{n}(t)$ the solution to :

$$
(I N T)_{n} \quad \mathcal{T}_{n}(v)=v
$$

Proposition 4 Let $T<T\left(u_{0}\right)$, if $n$ is sufficiently large, then the solutions to $(I N T)_{n}$ exist on $[0 ; T]$ and $u_{n} \rightarrow u$ in $\mathcal{C}\left(0 ; T ; H^{s}\right) \cap L^{q}\left(0 ; T ; W^{k+s, \beta}\right)$.

Proof : By standard arguments, it is sufficient to prove a local version.

Now the calculations of section 3.2 show that there exists $\tilde{T}>0$ depending only on $\left|u_{0}\right|_{H^{s}}$ such that the $\left(\mathcal{T}_{n}\right)$ are contraction in the ball of radius $R$ in $L^{\infty}\left(0 ; \tilde{T} ; H^{s}\right) \cap L^{q}\left(0 ; \tilde{T} ; W^{s+k, \beta}\right)$ for the norm of $L^{q}\left(0 ; \tilde{T} ; L^{\beta}\right)$; the rate of contraction being $1 / 2$. The continuous dependence of the fixed point follows by standard arguments.

\subsection{Equivalence between the integral equation and the partial differential equation}

In order to finish the proof of Theorem 1, we need to show :

Proposition 5 Let $u \in \mathcal{C}\left([0 ; T] ; H^{s}\right)$, $u$ satisfies (1) if and only if $u$ satisfies (INT). 
Proof : Let $u \in \mathcal{C}\left([0 ; T] ; H^{s}\right)$, then $f_{j}(u) \in L^{\beta^{\prime}} \hookrightarrow H^{-s}$ by (H3), so that by $(\mathrm{H} 2) \mathcal{L}_{j}\left(f_{j}(u)\right) \in H^{-s-l}$.

Next we need :

Lemma 2 i) if $u_{0} \in H^{\alpha}$ then $W_{0}(t) u_{0} \in \mathcal{C}\left([0 ; T] ; H^{\alpha}\right) \cap \mathcal{C}^{1}\left([0 ; T] ; H^{\alpha-m}\right)$ and

$$
\begin{gathered}
i \frac{\partial}{\partial t}\left(W_{0}(t) u_{0}\right)+L\left(W_{0}(t) u_{0}\right)=0, \\
W_{0}(0) u_{0}=u_{0} .
\end{gathered}
$$

ii) If $f \in L^{1}\left(0 ; T ; H^{\alpha}\right)$ then

$$
\Lambda f \equiv-i \int_{0}^{t} W_{0}(t-s) f(s) d s \in \mathcal{C}\left([0 ; T], H^{\alpha}\right) \cap A C\left([0 ; T] ; H^{\alpha-m}\right)
$$

and

$$
\begin{gathered}
\frac{i \partial}{\partial t}(\Lambda f)+L(\Lambda f)=f \\
\Lambda f(0)=0 .
\end{gathered}
$$

iii) if $v, f \in L^{1}\left(0 ; T ; H^{\alpha}\right)$ satisfy

$$
i v_{t}+L v=f
$$

then $v(0) \in H^{\alpha-m}$ exists and

$$
v=W_{0}(t) v(0)-i \int_{0}^{t} W_{0}(t-s) f(s) d s .
$$

These are classic tools for unitary operators and we shall omit the proof of these results. Now since $\mathcal{L}_{j}\left(f_{j}(u)\right) \in H^{-s-l}$ and $u \in \mathcal{C}\left([0 ; T] ; H^{s}\right)$, Proposition 5 follows from Lemma 2.

The proof of Theorem 1 is now complete.

\section{Appendix}

The aim of this Appendix is to prove the following theorem :

Theorem 3 Let $s>0, F \in \mathcal{C}^{r}\left(\mathbb{R}^{2}\right)$ with $r \geq[s]+1$. Suppose that $\exists \sigma \geq r-1$ such that $\forall_{j}$ with $|j| \leq r$,

$$
\left|D^{j} F(\xi)\right| \leq C|\xi|^{\sigma+1-|j|}, \quad \forall \xi \in \mathbb{R}^{2} .
$$

Then :

$$
|F(u)|_{W^{s, \beta}} \leq C|u|_{L^{z}}^{\sigma}|u|_{W^{s, p},}
$$

with

$$
\begin{gathered}
\frac{1}{\beta}=\frac{\sigma}{z}+\frac{1}{p}, 1<\beta, p<\infty \\
1<z \leq+\infty
\end{gathered}
$$


In [8], Y. Meyer shows that if $F \in \mathcal{C}^{\infty}\left(\mathbb{R}^{2}\right)$ with $F(0)=0$ and $u \in W^{s, p} \cap$ $L^{\infty}$ then $F(u) \in W^{s, p}$. We shall extend his proof to the case $W^{s, p} \cap L^{z}$, and we derive the inequality of Theorem 3 .

Proof : We take a radial nonincreasing function $\varphi \in \mathcal{D}\left(\mathbb{R}^{n}\right)$ such that $\varphi(\xi)=$ 1 for $|\xi| \leq 1 / 2$ and $\varphi(\xi)=0$ if $|\xi| \geq 1$. We denote by $S_{k}(f)=f_{k}$ the partial sum defined by :

$$
\widehat{S_{k}(f)}=\varphi\left(\frac{\xi}{2^{k}}\right) \hat{f}
$$

and by $\Delta_{k}(f)$ the dyadic block : $\Delta_{k}(f)=S_{k+1}(f)-S_{k}(f)$, i.e.

$$
\widehat{\Delta_{k}(f)}=\psi\left(\frac{\xi}{2^{k}}\right) \hat{f} \text { with } \psi(\xi)=\varphi\left(\frac{\xi}{2}\right)-\varphi(\xi) .
$$

The spectrum of $\Delta_{k}(f)$ is inclued in $\Gamma_{k}=\left\{\xi \in \mathbb{R}^{n} / \frac{2^{k}}{2} \leq|\xi| \leq 2.2^{k}\right\}$ and $f=S_{0}(f)+\Delta_{0}(f)+\cdots+\Delta_{k}(f)+\cdots$

We recall the characterization of $W^{s, p}=\left\{f \in S^{\prime} /(1-\Delta)^{s / 2} f \in L^{p}\right\}$ for $1<$ $p<\infty$. Then $f \in W^{s, p}$ if and only if $S_{0}(f) \in L^{p}$ and $\left(\sum_{k=0}^{+\infty} 4^{k s}\left|\Delta_{k} f\right|^{2}\right)^{1 / 2} \in L^{p}$. Moreover $\left\|S_{0}(f)\right\|_{L^{p}}+\left\|\left(\sum_{k=0}^{\infty} 4^{k s}\left|\Delta_{k}(f)\right|^{2}\right)^{1 / 2}\right\|_{L^{p}}$ and $\left\|(I-\Delta)^{1 / 2} f\right\|_{L^{p}}$ are two equivalent norms on $W^{s, p}$.

We first we have :

Lemma $3 \exists C$ independent of $k$ and $f$ such that:

$$
\begin{gathered}
\forall \alpha \in N^{n}\left|\partial^{\alpha} f_{k}\right|_{L^{p}} \leq C 2^{k|\alpha|}|f|_{L^{p}}, \\
\frac{1}{C} 2^{k q}\left|\Delta_{k}(f)\right|_{L^{p}} \leq \sum_{|\alpha|=q}\left|\partial^{\alpha} \Delta_{k}(f)\right|_{L^{p}} \leq C 2^{k q}\left|\Delta_{k}(f)\right|_{L^{p}}, \\
1<p<+\infty .
\end{gathered}
$$

Proof : For $1<p<\infty$, it is the application of Hörmander's Multiplier's Theorem. For the case $p=\infty$, see Alinhac - Gérard [1].

\section{Proof of the Theorem :}

We write

$$
f=S_{0}(f)+\sum_{k=0}^{+\infty} \Delta_{k}(f)
$$

and

$$
F(f)=F\left(f_{0}\right)+\sum_{k=0}^{+\infty}\left[F\left(f_{k+1}\right)-F\left(f_{k}\right)\right] .
$$

We estimate each term :

i) The term $F\left(f_{0}\right)$ :

$*\left|F\left(f_{0}\right)\right| \leq C\left|f_{0}\right|^{\sigma+1}$, Lemma 3 implies $\left|f_{0}\right|_{L^{p}} \leq C|f|_{L^{p}}$ and $\left|f_{0}\right|_{L^{z}} \leq C|f|_{L^{z}}$, so that

$$
\left|F\left(f_{0}\right)\right|_{L^{\beta}} \leq C\left|f_{0}\right|_{L^{z}}^{\sigma}\left|f_{0}\right|_{L^{p}}
$$


* We estimate now :

$$
\partial^{\alpha} F\left(f_{0}\right)=\sum_{\gamma+\cdots+\gamma_{q}=\alpha}\left(\partial^{|\alpha|} F\right)\left(f_{0}\right) \partial^{\gamma_{1}} f_{0} \cdots \partial^{\gamma_{a}} f_{0} \text { with }|\alpha|<[s+1] .
$$

Then by Lemma 3, $\left|\partial^{\gamma_{1}} f_{0}\right|_{L^{p}} \leq C\left|f_{0}\right|_{L^{p}}$ and for $i>1,\left|\partial^{\gamma_{i}} f_{0}\right|_{L^{z}} \leq C\left|f_{0}\right|_{L^{z}}$, so that

$$
\left|\partial^{\alpha} F\left(f_{0}\right)\right|_{L^{\beta}} \leq C|f|_{L^{z}}^{\sigma}|f|_{L^{p}}, \forall|\alpha|<[s+1] .
$$

With (8) and (9) we obtain

$$
\left|F\left(f_{0}\right)\right|_{W^{s, \beta}} \leq C|f|_{L^{z}}^{\sigma}|f|_{L^{p}}
$$

ii) The terms $F\left(f_{k+1}\right)-F\left(f_{k}\right)$ :

$$
F\left(f_{k+1}\right)-F\left(f_{k}\right)=\Delta_{k}(f) \int_{0}^{1} F^{\prime}\left(f_{k}+t \Delta_{k}(f)\right) d t=\Delta_{k}(f) m_{k} .
$$

- We first suppose that the spectrum of $m_{k}(x) \Delta_{k}(f)(x)$ is include in $\{|\xi| \leq$ $\left.100.2^{k}\right\}$ and we consider $h_{k}(x)=m_{k}(x) \Delta_{k}(f)(x)$.

Let $\sigma(x)=\left(\sum_{j} 4^{j s}\left|\Delta_{j}\left(\sum_{k} h_{k}\right)\right|^{2}\right)^{1 / 2}$.

\section{Lemma 4}

$$
|\sigma(x)|_{L^{\beta}} \leq C||\left(\left.\sum_{k} m_{k}(x) \Delta_{k}(f)\right|^{2} 4^{k s}\right)^{1 / 2} \|_{L^{\beta}}
$$

Proof : See Meyer [8].

To continue, we need

Lemma 5 For any $f$,

$$
\forall|N|<\sigma+1,\left|\partial^{N} m_{k}(x)\right| \leq C|M(f)(x)|{ }^{\sigma} 2^{|N| k},
$$

where $M(f)$ is the maximal function of $f$.

The proof of Lemma 5 depends on

Lemma 6 Let $\varphi \in S$ and $\varphi_{\varepsilon}(x)=\frac{1}{\varepsilon^{n}} \varphi\left(\frac{x}{\varepsilon}\right)$, then

$$
\sup _{\varepsilon>0}\left|\left(f * \varphi_{\varepsilon}\right)(x)\right| \leq C M(f)(x),
$$

for $f \in L^{p}, 1 \leq p \leq+\infty$.

For the proof of Lemma 6, see Stein [10] p. 62-64.

We can now prove Lemma 5 :

$$
\left|\partial^{N} m_{k}(x)\right| \leq C \sum_{\alpha_{i}+\cdots+\alpha_{q}=N}\left(D^{N+1} F\right)\left(f_{k}\right)\left|\partial^{\alpha_{1}} f_{k}\right| \cdots\left|\partial^{\alpha_{q}} f_{k}\right|
$$

and by Lemma $6,\left|f_{k}(x)\right| \leq C M(f)(x)$;

moreover

$$
\begin{aligned}
& \partial^{\alpha_{i}} f_{k}(x)=\Im^{-1}\left(\xi^{\alpha_{i}} \varphi\left(\frac{\xi}{2^{k}}\right) \hat{f}\right), \\
& =2^{\left|\alpha_{i}\right| k} \Im^{-1}\left(\left(\frac{\xi}{2^{k}}\right)^{\alpha_{i}} \varphi\left(\frac{\xi}{2^{k}}\right) \hat{f}\right) .
\end{aligned}
$$

We apply Lemma 6 again with the function $\Im^{-1}\left(\left(\frac{\xi}{2^{k}}\right)^{\alpha_{i}} \varphi\left(\frac{\xi}{2^{k}}\right)\right)$; we obtain $\left|\partial^{\alpha_{i}} f_{k}(x)\right| \leq C 2^{\left|\alpha_{i}\right| k} C M(f)(x)$ and Lemma 5 follows. 
Now

$$
\begin{gathered}
|\sigma(x)|_{L^{\beta}} \leq\left.\left. C|| M(f)(x)\right|^{\sigma}\left(\sum_{k}\left|\Delta_{k}(f)\right|^{2} 4^{k s}\right)^{1 / 2}\right|_{L^{\beta}}, \\
|\sigma(x)|_{L^{\beta}} \leq C|M(f)(x)|_{L^{z}}^{\sigma}|f|_{W^{s, p}} .
\end{gathered}
$$

- General case : we decompose $m_{k}(x)$ with the following partition of 1 :

$$
1=\varphi\left(\frac{\xi}{100.2^{k}}\right)+\sum_{m=0}^{+\infty} \psi\left(\frac{\xi}{100.2^{k+m}}\right),
$$

i.e. $m_{k}(x)=q_{k}(x)+\sum_{m=0}^{\infty} P_{k, m}(x)$.

The spectrum of $q_{k}$ is inclued in $\left\{|\xi| \leq 1002^{k}\right\}$, so that the proof of the preceding case applies to $q_{k}$ and thanks to (11)

$$
\left|\sum_{k} q_{k}(x) \Delta_{k}(f)\right|_{W^{s, \beta}} \leq C|f|_{L^{z}}^{\sigma}|f|_{W^{s, p}} .
$$

Now the spectrum of $P_{k, m}$ is inclued in the ring

$$
\left\{\frac{100}{2} 2^{k+m} \leq|\xi| \leq 2.100 .2^{k+m}\right\}
$$

We define $l_{m}=\sum_{k=0}^{+\infty} P_{k, m}(x) \Delta_{k}(f)(x)$. The spectrum of $P_{k, m} \Delta_{k}(f)$ is inclued in the ring $\left\{\frac{100}{3} 2^{k+m} \leq|\xi| \leq 3.100 .2^{k+m}\right\}$, these rings taken 5 by 5 are disjoint and we can apply the Littlewood - Paley theory on

$$
S_{r}(x)=\sum_{k \in 5 N_{+r}} P_{k, m} \Delta_{k}(f):
$$

We estimate

$$
\left|S_{r}\right|_{W^{s, \beta}}=\|\left(\sum_{k}\left|P_{k, m}\right|^{2}\left|\Delta_{k}(f)\right|^{2} 4^{(k+m) s}\right)^{1 / 2}||_{L^{\beta}},
$$

but

$$
P_{k, m}(x)=\int e^{i x \cdot \xi} \psi\left(\frac{\xi}{100.2^{k+m}}\right) \widehat{m_{k}}(\xi) d \xi
$$

We introduce a partition of unity on the sphere $S^{n-1},\left(\chi_{p}\right)_{p=1 . . n}$, such that on $\operatorname{supp} \chi_{p}, \xi_{p} \neq 0$. We extend $\chi_{p}$ into $R^{n} /\{0\}$ by $\chi_{p}(\xi)=\chi_{p}\left(\frac{\xi}{|\xi|}\right)$.

Let $N=[s]+1$, we obtain

$$
\begin{gathered}
P_{k, m}(x)=\sum_{p=1}^{n} \int e^{i x \cdot \xi} \psi\left(\frac{\xi}{100.2^{k+m}}\right) \widehat{m_{k}}(\xi) \chi_{p}(\xi) d \xi \\
=\sum_{p=1}^{n} \int e^{i x \cdot \xi} \frac{1}{\xi_{p}^{N}} \psi\left(\frac{\xi}{100.2^{k+m}}\right) \xi_{p}^{N} \widehat{m_{k}}(\xi) \chi_{p}(\xi) d \xi \\
=\sum_{p=1}^{n} \frac{2^{-(k+m) N}}{100} \int e^{i x \cdot \xi} 100 \frac{2^{(k+m) N}}{\xi_{p}^{N}} \psi\left(\frac{\xi}{100.2^{k+m}}\right) \frac{\partial^{N_{m}}}{\partial x_{p}^{N}}(\xi) \chi_{p}\left(\frac{\xi}{100.2^{k+m}}\right) d \xi
\end{gathered}
$$


since $\chi_{p}$ is homogeneous of degre 0 ,

$$
\sum_{p=1}^{n} \frac{2^{-(k+m) N}}{100} \mathcal{F}^{-1}\left(\left(\frac{\psi}{\xi_{p}^{N}} \chi_{p}\right)\left(\frac{\xi}{100.2^{k+m}}\right)\right) * \frac{\partial^{N} m_{k}}{\partial x_{p}^{N}} .
$$

So that

$$
\left|P_{k, m}(x)\right| \leq \sum_{p=1}^{n} C \frac{2^{-(k+m) N}}{100} M\left(\frac{\partial^{N} m_{k}}{\partial x_{p}^{n}}\right)(x),
$$

by lemma 6 .

It follows that

$$
\left|P_{k, m}(x)\right| \leq C \frac{2^{-(k+m) N}}{100} M\left(|M(f)(x)|^{\sigma}\right) 2^{k N}
$$

by lemma 5 ,

$$
\leq C 2^{-m N} M\left(|M(f)(x)|^{\sigma}\right)
$$

So that

$$
\begin{gathered}
\left|S_{r}\right|_{W^{s, \beta}} \leq \|\left. M\left(|M(f)(x)|^{\sigma}\right)\left(\sum_{k}\left|\Delta_{k}(f)\right|^{2} 4^{k s}\right)^{1 / 2} 2^{-m(N-s)}\right|_{L^{\beta}} \\
\leq C|f|_{L^{z}}^{\sigma}|f|_{W^{s, p}} 2^{-m(N-s)}
\end{gathered}
$$

We obtain $\left|l_{m}\right|_{W^{s, \beta}} \leq C 2^{-m[N-s]}|f|_{L^{z}}^{\sigma}|f|_{W^{s, p}}$ and the serie $\sum_{m} l_{m}$ converges normaly in $W^{s, \beta}$, with

$$
\left|\sum_{m} l_{m}\right|_{W^{s, \beta}} \leq C|f|_{L^{z}}^{\sigma}|f|_{W^{s, p}}
$$

(10), (12) and (13) together lead to the estimate of Theorem 3.

\section{References}

[1] S. Alinhac, P. Gérard : Opérateurs pseudo-différentiels et théorème de Nash-Moser. Interéditions - Editions du CNRS. 1991.

[2] M.Christ, M.Weinstein : Dispersion of small amplitude solutions of the generalized KdV equation. Journ. Funct. Anal. 100, 87-109, 1991.

[3] R.T. Glassey : On the blowing up solutions to the Cauchy problem for nonlinear Schrödinger equations, J. Math. Phys. 18 (1977) 1794-1797.

[4] C.Kenig, G.Ponce, L.Vega : Oscillatory integrals and regularity of dispersive equations, Indiana Univ. Math. Journ. vol $40 N^{0} 1,1991$.

[5] C.Kenig, G.Ponce, L.Vega : Well-posedness and scattering results for the generalized $\mathrm{KdV}$ equation via the contraction principle. To appear. 
[6] C.Kenig, G.Ponce, L.Vega : Small solutions to nonlinear Schrödinger equations. To appear.

[7] S.Klainerman, G.Ponce : Global, small solution to nonlinear evolution equations. Comm. Pure. Appl. Math. vol36, 133-141, 1983.

[8] Y. Meyer : Remarques sur un théorème de J.M. Bony. Suppl. di Rendiconti del circ. Mat. di Palermo, Atti del Seminario di Analisa Armonica, Pisa, Série $2, N^{0} 1,1981$.

[9] J.C. Saut : Sur quelques généralisations de l'équation de Korteweg de Vries. J. Math. Pures et Appl. 58, 1979, 21-61.

[10] E. Stein : Singular integrals and differentiability properties of functions. Princeton University Press 1970.

[11] Torschinsky : Real variable methods in Harmonic Analysis. Academic Press 1986. 